\title{
Pratiques phytosanitaires des producteurs de tomates du Burkina Faso et risques pour la santé et l'environnement
}

\author{
Diakalia Son ${ }^{1,3, *}$, Irénée Somda ${ }^{1}$, Anne Legreve ${ }^{2}$ et Bruno Schiffers ${ }^{3}$ \\ ${ }^{1}$ Institut du développement rural, université polytechnique de Bobo-Dioulasso, laboratoire de phytopathologie, Sise à Nasso, \\ 01 BP 1091, Bobo-Dioulasso 01, Burkina Faso \\ ${ }^{2}$ Faculté des bio-ingénieurs, Earth and Life Institute, université catholique de Louvain, laboratoire de phytopathologie, \\ Louvain-La-Neuve, Belgique \\ ${ }^{3}$ Gembloux Agro-Bio Tech, université de Liège, laboratoire de phytopharmacie, passage des Déportés 2, 5030 Gembloux, Belgique
}

\begin{abstract}
Résumé - Pour évaluer les pratiques phytosanitaires des maraîchers du Burkina Faso, des enquêtes ont été réalisées en 2015 et 2016 auprès de 316 producteurs dans les zones Ouest et Nord du pays. Seuls $9 \%$ d'entre eux ont reçu une formation en protection des végétaux. Quatre-vingt-dix pour cent des pesticides utilisés sont achetés sur les marchés locaux sans garantie de conformité et de qualité. La lambdacyhalothrine de la famille des pyréthrinoïdes est la substance active la plus utilisée. L'utilisation sur tomate des pesticides formulés pour le coton a fortement progressé entre 2015 et 2016 (71\%). Les doses utilisées par les producteurs sont supérieures à celles recommandées. Plus de $70 \%$ des maraîchers n'observent aucune mesure de protection adéquate depuis la préparation de la bouillie jusqu'à la fin des traitements. Le respect de délais sans traitement avant récolte dépend plus de l'état sanitaire des parcelles que des recommandations. Les contenants vides sont abandonnés sur les lieux de traitement par $53 \%$ des répondants. Les pratiques phytosanitaires des maraîchers du Burkina Faso sont donc préoccupantes et potentiellement nuisibles pour la santé des agriculteurs et des consommateurs, et pour l'environnement.
\end{abstract}

Mots clés : cultures maraîchères / tomate / pesticide / pratiques phytosanitaires / risques chimiques

\begin{abstract}
Phytosanitary practices of tomato growers in Burkina Faso and risks for health and the environment. To assess the phytosanitary practices of tomato growers in Burkina Faso, surveys were carried out in 2015 and 2016 among 316 producers in the West and North zones of the country. Ninety percent of pesticides used by producers are purchased in local markets without guarantee of conformity or quality. Lambda-cyhalothrin of the pyrethroids family was the most used active substance. The use for tomato crop of cotton pesticides increased strongly between 2015 and $2016(+71 \%)$. The doses used by producers are higher than the recommended ones. More than $70 \%$ of the market gardeners do not observe any measure of adequate protection from the preparation of the mixture until the end of the treatments. The respect of preharvest interval depends mostly of the crop health status and does not follow the recommendations. Empty containers of pesticides are left in fields by $53 \%$ of the farmers. These results show that the phytosanitary practices of tomato producers are unsuitable and potentially harmful to the health of farmers and consumers and to the environment.
\end{abstract}

Keywords: market gardening / tomato / pesticides / phytosanitary practices / chemical risks

\section{Introduction}

$\mathrm{Au}$ Burkina Faso, la tomate est la deuxième plus importante culture maraîchère, après l'oignon, avec une production estimée à plus de 10000 tonnes en 2014 (FAOSTAT, 2016). Cependant, au cours de ces dernières années, on a assisté à une baisse des

\footnotetext{
*Auteur de correspondance : sondiakalia@yahoo.fr
}

rendements passés de 11,3 tonnes/ha en 2010 à 9,7 tonnes/ha en 2014 (FAOSTAT, 2016). Ces baisses de rendement sont principalement dues à un complexe de bioagresseurs dont les principaux sont Ralstonia solanacearum (Smith) ; Fusarium oxysporum (Scheltdl) ; Bemisia tabaci (Genn) ; Helicoverpa armigera (Hub) et Tuta absoluta (Meyrick).

Pour faire face à ces bioagresseurs, l'utilisation des pesticides chimiques de synthèse est la solution la plus utilisée. Cependant, plusieurs études au Burkina Faso (IFDC, 2007 ; 


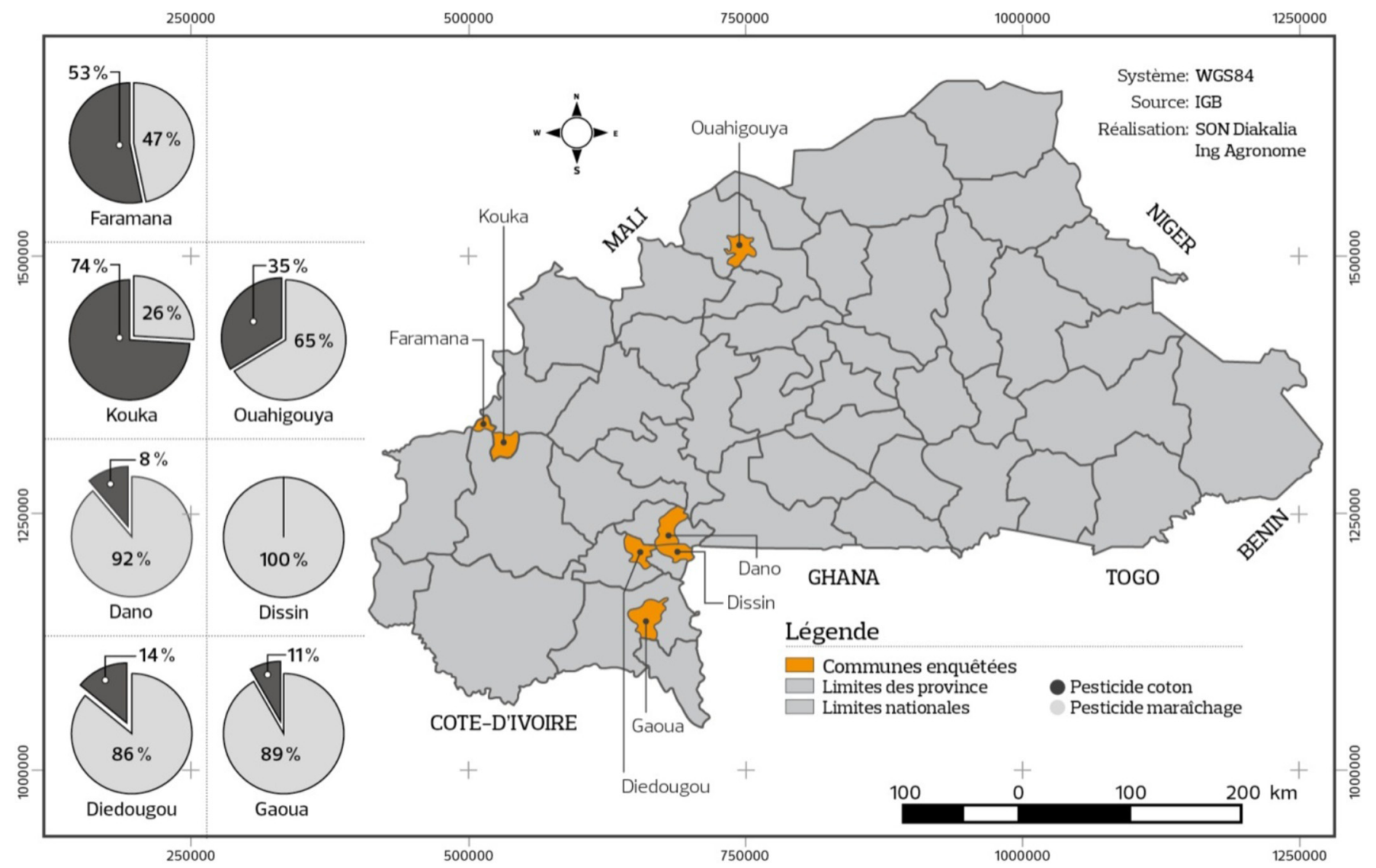

Fig. 1. Répartition spatiale des sept communes d'enquêtes et taux d'utilisation des pesticides adaptés au coton en maraîchage en 2015 et 2016. Fig. 1. Spatial distribution of the seven survey communes and use of cotton pesticides in market gardening in 2015 and 2016.

Toé, 2010 ; Naré et al., 2015) ont souligné l'existence de mauvaises pratiques phytosanitaires : non-respect des doses prescrites, non-respect des règles de protection et d'hygiène conseillées lors des traitements, mauvaise gestion des emballages vides des pesticides. Les conséquences en sont l'intoxication des agriculteurs et des consommateurs, la pollution de l'environnement et la sélection de souches de bioagresseurs résistantes aux pesticides (Cissé et al., 2006 ; Toé, 2010 ; Ahouangninou et al., 2011 ; Ngom et al., 2012 ; Gnankiné et al., 2013 ; Roditakis et al., 2015; Lehmann et al., 2016a).

Cette étude a donc été menée auprès des producteurs de tomates de l'Ouest et du Nord du Burkina Faso pour comprendre les motifs qui déterminent l'emploi des produits chimiques dans la production de tomates et évaluer les pratiques phytosanitaires de ces producteurs en termes d'impacts potentiels sur la santé humaine et sur l'environnement.

\section{Méthodologie d'enquête}

L'enquête a été menée dans sept communes (Faramana, Kouka, Dano, Dissin, Diébougou, Gaoua et Ouahigouya) (Fig. 1), auprès de 316 producteurs de tomate choisis de façon aléatoire sur 21 sites. Deux critères ont guidé le choix des sites d'étude : l'importance du site dans la production nationale de tomate et son emplacement géographique (la proximité avec les frontières pouvant faciliter l'introduction frauduleuse de pesticides non autorisés au Burkina Faso). L'étude a été conduite en 2015 et 2016 pendant la grande période de production maraîchère qui va de novembre à avril (saison sèche). Les entretiens avec les producteurs ont porté sur leur niveau d'instruction, les cultures pratiquées, les principaux problèmes phytosanitaires rencontrés, les méthodes de lutte utilisées, les produits phytosanitaires utilisés, le dosage, les moyens de protection utilisés, la gestion des emballages et leurs connaissances du risque chimique.

Les données collectées ont été saisies et traitées à l'aide des logiciels Excel et IBM SPSS.23.

\section{Résultats}

\subsection{Caractéristiques sociodémographiques des producteurs de tomate}

Les producteurs de tomate rencontrés au cours de ces deux années d'enquête sont en grande majorité des hommes (92\%) âgés de 20 à 50 ans. Soixante-douze pour cent d'entre eux n'ont reçu aucune instruction et seulement $9 \%$ ont reçu une formation en protection des végétaux (Tab. 1). Plus de $65 \%$ des interviewés ont déclaré n'avoir reçu aucun appui-conseil de la part des agents de vulgarisation agricole dans le domaine du maraîchage. 
Tableau 1. Statut social des producteurs de tomate au Burkina Faso (enquête réalisée dans sept communes : Faramana, Kouka, Dano, Dissin, Diébougou, Gaoua et Ouahigouya) en 2015 et 2016.

Table 1. Social status of tomato growers in Burkina Faso (survey conducted in seven communes: Faramana, Kouka, Dano, Dissin, Diébougou, Gaoua and Ouahigouya) in 2015 and 2016.

\begin{tabular}{lll}
\hline & Statut social des & Valeurs en \\
& producteurs de tomate & pourcentage (\%) \\
\hline \multirow{3}{*}{ Sexe } & Homme & 92 \\
& Femme & 8 \\
Niveau d'instruction & Aucune & 72 \\
\multirow{2}{*}{ Formation en protection } & Primaire & 21 \\
des végétaux & Secondaire & 7 \\
& Aucun & 91 \\
& Oui & 9 \\
\hline
\end{tabular}

\subsection{Pression des bioagresseurs et des facteurs abiotiques sur la tomate}

Les principaux ravageurs de la tomate signalés par les producteurs ou constatés sur les sites d'enquête sont H. armigera, T. absoluta, B. tabaci, Tetranychus urticae (Koch), Aculops lycopersici (Massee) et Meloidogyne spp. Les principales maladies sont la fusariose, l'alternariose, le flétrissement bactérien, les fontes des semis et le virus responsable de l'enroulement des feuilles (Tomato Yellow Leaf Curl Virus). De plus, la nécrose apicale causée, soit par une carence en calcium, soit par stress hydrique (mauvaise répartition des irrigations), est fréquemment observée chez les producteurs ( $45 \%$ ), avec un impact sur la qualité des fruits.

\subsection{Pratiques phytosanitaires observées et risque pour la santé et l'environnement}

Pour lutter contre ces bioagresseurs, la lutte chimique au moyen des pesticides de synthèse est la plus pratiquée par les producteurs. Ils achètent ces produits sur les marchés locaux et $90 \%$ des pesticides utilisés sont ainsi achetés sans garantie de conformité et de qualité. Les substances actives les plus utilisées (Tab. 2) au cours de ces enquêtes sont la lambdacyhalothrine (67\%), l'acétamipride (50\%) et la cyperméthrine $(37 \%)$. Parmi les familles chimiques, les plus utilisées sont les pyréthrinoïdes $(62,5 \%)$ et les organophosphorés $(37,5 \%)$. Les biopesticides sont faiblement utilisés $(5 \%)$. Le taux d'utilisation sur culture de tomate de pesticides normalement réservés au coton était de $35 \%$ en 2015 et de $60 \%$ en 2016 , soit une progression de $71 \%$. Ces insecticides adaptés au coton sont plus fréquemment recensés dans les communes de Faramana et de Kouka (Fig. 1), qui sont situées dans les grandes zones de production cotonnière des HautsBassins et de la Boucle du Mouhoun. Le dosage, la préparation et l'application des pesticides sont réalisés soit par les propriétaires des parcelles $(78 \%)$, soit par leurs enfants $(7 \%)$, soit par leurs employés $(14,5 \%)$. Les femmes qui réalisent des traitements phytosanitaires ne représentent que $0,5 \%$ du total et principalement dans les communes de Faramana et de Kouka. Les doses de pesticides sont mesurées
Tableau 2. Types de pesticides utilisés par les producteurs de tomate dans sept communes du Burkina Faso (Faramana, Kouka, Dano, Dissin, Diébougou, Gaoua et Ouahigouya) en 2015 et 2016.

Table 2. Types of pesticides used by tomato growers in seven communes of Burkina (Faramana, Kouka, Dano, Dissin, Diébougou, Gaoua and Ouahigouya) in 2015 and 2016.

\begin{tabular}{lll}
\hline & $\begin{array}{l}\text { Statut des pesticides } \\
\text { utilisés }\end{array}$ & $\begin{array}{l}\text { Valeurs en } \\
\text { pourcentage (\%) }\end{array}$ \\
\hline & Lambda-cyhalothrine & 67 \\
Substances actives & Acétamipride & 50 \\
les plus utilisées & Cyperméthrine & 37 \\
& Profenofos & 25 \\
Familles chimiques & Pyréthrinoïde & 63 \\
les plus utilisées & Organophosphoré & 38 \\
& Néonicotinoïde & 21 \\
\hline
\end{tabular}

à l'aide des bouchons des emballages et cela de façon approximative, surtout en ce qui concerne les formulations en poudre (WP) qui nécessiteraient une pesée. Par conséquent, les dosages varient fortement d'un maraîcher à un autre, d'un traitement à un autre et sont dans la majorité des cas supérieurs à la dose recommandée sur l'étiquette. En moyenne, $26 \%$ des producteurs mélangent plusieurs préparations commerciales pour obtenir des concentrations qui leur conviennent. Vingt et un pour cent conservent leurs préparations pendant plusieurs jours pour effectuer des traitements successifs ; à chaque application, une petite quantité de la préparation est diluée à nouveau avant de traiter. Par ailleurs, $35 \%$ des producteurs reconditionnent leurs produits dans un autre contenant (Fig. 2). Les emballages vides sont le plus souvent abandonnés sur les lieux du traitement (53\%), parfois jetés, enfouis dans le sol ou brûlés à l'air libre (Fig. 3).

Les fréquences d'application varient d'un producteur à un autre, mais dans la majorité des cas elles sont d'une fois par semaine. Le nombre moyen de traitements effectués par cycle de production de tomate est de dix.

Les équipements de protection individuelle (EPI) se limitent au port de tenues ordinaires perméables aux pesticides (Fig. 4). Ces tenues sont composées d'un pantalon ou d'une culotte ordinaires et d'une chemise à manches longues ou courtes. Les principaux arguments avancés pour justifier cette « non-protection adéquate » sont la non-disponibilité des EPI conformes, le coût d'achat trop élevé de ces EPI et la gêne (du fait de la chaleur) occasionnée par le port de ces équipements de protection pendant l'application.

La plupart des producteurs rencontrés ont rapporté avoir ressenti, après utilisation des pesticides, des effets des traitements sur leur santé tels que des irritations de la peau (26\%), des bouffées de chaleur (19\%), des écoulements du nez et une toux $(15 \%)$, des affections oculaires $(8 \%)$ et des céphalées (4\%) (Fig. 5). Pour lutter contre ces effets néfastes ou pour éviter d'éventuels effets secondaires, plus de $60 \%$ des interviewés déclarent boire du lait après les traitements afin de se désintoxiquer.

En ce qui concerne le délai d'attente avant la récolte (DAR), intervalle de temps entre le dernier traitement autorisé 


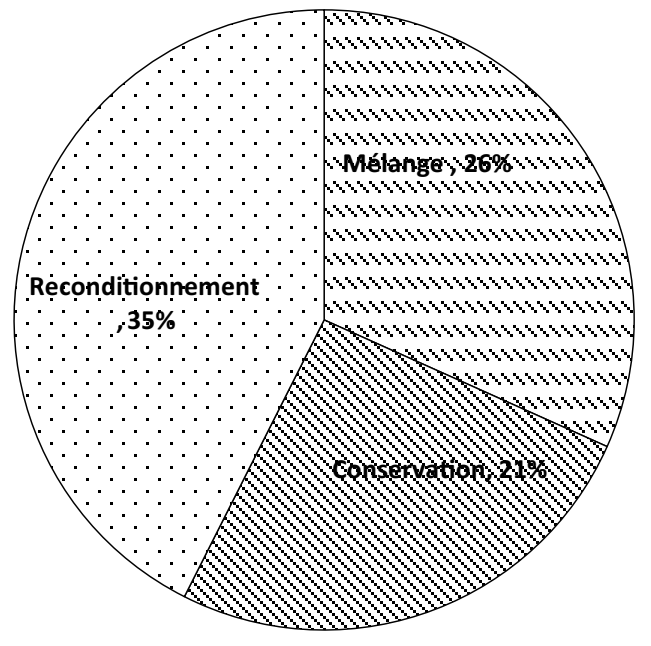

Fig. 2. Proportion des producteurs de tomate conservant leurs bouillies et pratiquant le reconditionnement des pesticides dans les sept communes étudiées en 2015 et 2016 au Burkina Faso.

Fig. 2. Ratio of tomato growers who store their mixture and repackage their pesticides in the seven communes surveyed in 2015 and 2016 in Burkina Faso.

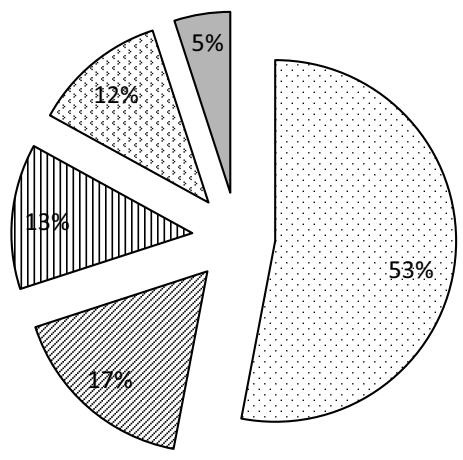

๑ Abandonne dans le champ

$\square$ Jette dans puits perdus

四 Enfouit dans le sol

๑r Réutilise

$\square$ Brûle

Fig. 3. Modes de gestion des emballages vides de pesticides par les producteurs de tomate dans les sept communes étudiées en 2015 et 2016 au Burkina Faso.

Fig. 3. Management of empty packaging of pesticides by tomato growers in the seven communes surveyed in 2015 and 2016 in Burkina Faso.

et la récolte, son respect varie d'un producteur à un autre et est plus lié à l'état sanitaire des fruits à ce moment-là (absence de ravageurs) qu'aux recommandations d'emploi du produit phytosanitaire. Par exemple le DAR pour l'insecticide TITAN $25 \mathrm{EC}$ est de 7 à 15 jours, alors qu'il varie de 3 à 7 jours chez $53 \%$ des maraîchers.

\section{Discussion}

\subsection{Facteurs jouant sur l'utilisation des pesticides}

L'enquête montre un faible niveau d'instruction et de formation technique des producteurs. Cela ne favorise pas une bonne utilisation des produits phytosanitaires par méconnaissance des bioagresseurs et du produit adéquat à utiliser en fonction de la cible (Ahouangninou et al., 2011 ; Kanda et al., 2013 ; Wognin et al., 2013 ; Naré et al., 2015).

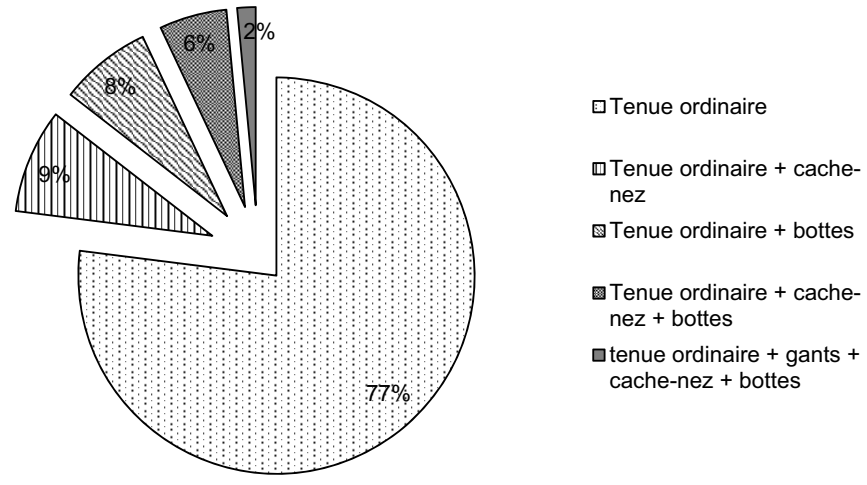

Fig. 4. Proportion des producteurs de tomate portant des équipements de protection individuelle (EPI) dans les sept communes étudiées en 2015 et 2016 au Burkina Faso.

Fig. 4. Ratio of tomato growers wearing personal protective equipment (PPE) in the seven communes surveyed in 2015 and 2016 in Burkina Faso.

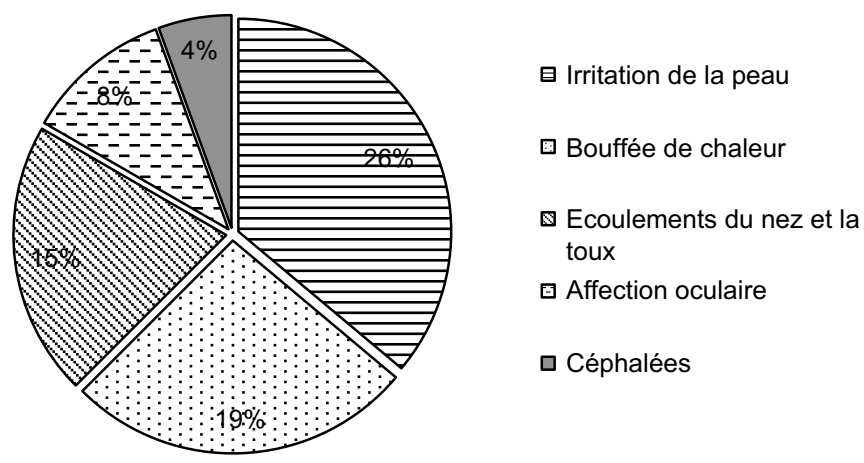

Fig. 5. Nature des affections ressenties par les producteurs interrogés après l'application des pesticides dans les sept communes étudiées en 2015 et 2016 au Burkina Faso.

Fig. 5. Nature of the affections experienced by producers interviewed after application of pesticides in seven communes surveyed in 2015 and 2016 in Burkina Faso.

Parmi les pesticides, la famille chimique des pyréthrinoïdes est la plus utilisée, alors que certains auteurs (Martin et al., 2005 ; Achaleke et Brévault, 2009 ; Abou-Yousef et al., 2010 ; Houndété et al., 2010 ; Gnankiné et al., 2013) ont signalé la résistance d'H. armigera et de $B$. tabaci (principaux ravageurs de la tomate au Burkina Faso) à cette famille chimique. Cette situation amène les producteurs à augmenter le nombre de traitements et les doses utilisées. La conservation de la préparation de pesticide pendant plusieurs jours pour effectuer des traitements entraîne sa dénaturation et sa faible efficacité, obligeant ainsi les producteurs à intensifier les traitements ou à augmenter les dosages.

L'insuffisance de formation et de suivi des maraîchers amène ces derniers à utiliser des produits inadéquats et à épandre des doses inadaptées. La majorité des pesticides utilisés par les maraîchers sont achetés sur les marchés locaux et sont d'origine étrangère (Ghana et Côte d'Ivoire). Cela constitue une infraction à la loi burkinabé sur les pesticides et montre l'insuffisance de contrôle de l'État sur la vente et sur l'utilisation des pesticides. 


\subsection{Les risques pour la santé publique et l'environnement}

L'utilisation des pesticides nécessite un minimum de connaissances théoriques et pratiques pour écarter tout risque sur la santé humaine et sur l'environnement (Cissé et al., 2006 ; Kanda et al., 2013 ; Wognin et al., 2013), alors que l'enquête montre un faible niveau d'instruction, de formation et de suivi des maraîchers. Cela contribue à augmenter le risque d'intoxication et de pollution de l'environnement. Les pesticides destinés au coton ne sont pas recommandés en maraîchage en raison de leur toxicité élevée, de leur forte concentration et de leur persistance sur la culture (Schiffers et Mar, 2011). En cas d'utilisation, il faudrait au moins respecter la dose efficace et les conditions d'utilisation. Mais ne sachant ni lire, ni écrire, ni réaliser de calculs, la plupart des producteurs ne peuvent comprendre les étiquettes rédigées en français (voire en anglais pour les produits venant du Ghana), ni respecter les instructions d'usage ou même interpréter les pictogrammes de sécurité.

Peu de producteurs interrogés se conforment aux règles d'hygiène au cours de l'utilisation des pesticides. Ce constat rejoint celui fait par Doumbia et Kwadjo (2009) en Côte d'Ivoire, par Tyagi et al. (2015) en Inde et par Belhadi et al. (2016) en Algérie. Cela explique les cas d'intoxication aiguë rapportés par les producteurs et également exposés par d'autres auteurs (Toé, 2010 ; Ahouangninou et al., 2011 ; Tarla et al., 2013, 2015 ; Lehmann et al., 2016b). Les effets possibles sur la santé sont d'autant plus préoccupants que l'on note la présence d'enfants et de femmes dans les parcelles où sont appliqués les pesticides. Diverses études ont signalé un accroissement du risque de maladies ou de malformation chez l'homme, en général lié aux pesticides (Multigner, 2005 ; FAO et OIT, 2013). Chez les femmes, les conséquences possibles d'une exposition lors de l'utilisation des pesticides sont l'avortement spontané et la prématurité et la malformation des nouveau-nés (Samuel et Saint-Laurent, 2001 ; Multigner, 2005).

Afin de protéger la santé des consommateurs, il est nécessaire de respecter les doses d'utilisation et surtout le DAR de chaque produit, de façon à éviter que les résidus de pesticides contenus dans les légumes traités ne dépassent les limites maximales en résidus. Cependant, les producteurs ignorent en général ce délai et arrêtent leur traitement non pas en fonction des recommandations propres à chaque produit phytosanitaire, mais plutôt en fonction de la présence ou non de bioagresseurs dans leur parcelle. Ce non-respect du DAR a été également constaté par d'autres auteurs (Doumbia et Kwadjo, 2009 ; Ahouangninou et al., 2011 ; Kanda et al., 2013).

L'intensification des traitements et le non-respect des doses recommandées, additionnés à la mauvaise gestion des emballages vides de pesticides (Doumbia et Kwadjo, 2009 ; Ahouangninou et al., 2011 ; Belhadi et al., 2016), engendrent des effets néfastes sur l'environnement. Naré et al. (2014) ont constaté une diminution significative de la déshydrogénase du sol suite à l'utilisation d'endosulfan, de deltaméthrine et de profenofos en production maraîchère au Burkina Faso. Lehmann et al. (2016a), soulignent la pollution de certains cours d'eau du Burkina Faso par les résidus de pesticides dans les zones maraîchères. Comme certaines retenues d'eau peuvent servir à l'élevage de poissons, on peut craindre un transfert des résidus de pesticides jusqu'à l'homme par la chaîne alimentaire, comme cela a été observé au Bénin à travers des tilapias dans des conditions similaires (Agbohessi et al., 2012).

\section{Conclusion et recommandations}

Les pesticides utilisés sont achetés dans la majorité des cas dans les marchés locaux sans garantie de conformité et de qualité. Les pyréthrinoïdes sont les plus utilisés, alors que plusieurs auteurs ont souligné la résistance des ravageurs concernés ( $B$. tabaci et $H$. armigera) à cette famille chimique. Cela va contribuer à intensifier les traitements et par conséquent à exposer encore les agriculteurs qui sont faiblement protégés, et les consommateurs (du fait du nonrespect des DAR) aux risques chimiques. Les déchets de pesticides qui sont abandonnés dans la nature, et l'utilisation de doses souvent plus fortes que celles recommandées contribuent à polluer l'environnement.

Pour favoriser une gestion rationnelle des pesticides et limiter leurs impacts sur la santé humaine et sur l'environnement au Burkina Faso, plusieurs actions devraient être menées :

- en premier lieu, il faudrait conduire des actions de formation des producteurs et des vendeurs de pesticides pour améliorer leur connaissance des ennemis des cultures et des règles d'utilisation des pesticides : préciser et expliquer les techniques de diagnostic, les consignes de base de sécurité et l'importance des équipements de protection ;

- en second lieu, il faudrait favoriser les contacts des producteurs avec les instituts de recherche et les services techniques du ministère chargé de l'agriculture, de manière à ce qu'ils puissent réagir de façon appropriée à l'apparition de nouveaux ravageurs, ou de nouveaux risques ;

- enfin, il serait très utile de proposer des actions permettant une réduction de l'utilisation des pesticides en agriculture par la promotion et la vulgarisation des méthodes alternatives basées sur l'utilisation de la lutte intégrée, même si cette voie n'est pas facile à suivre comme le montrent par exemple les difficultés d'application du plan Ecophyto en France (Guichard et al., 2017).

Remerciements. Cette étude a été réalisée avec le soutien financier de ARES-CDD (Projet PIC « Projet de renforcement des capacités de diagnostic et de gestion intégrée des problèmes phytosanitaires au Burkina Faso », UCL-ULgIDR / UPB).

\section{Références}

Abou-Yousef HM, Farghaly SF, Singab M, Ghoneim YF. 2010. Resistance to lambda-cyhalothrin in laboratory strain of whitefly Bemisia tabaci (Genn.) and cross-resistance to several insecticides. American Eurasian Journal Agriculture \& Environnement Sciences 7 (6): 693-696.

Achaleke J, Brévault T. 2009. Inheritance and stability of pyrethroid resistance in the cotton bollworm Helicoverpa armigera (Lepidoptera: Noctuidae) in Central Africa. Pest Management Science 137-141. 
Agbohessi TP, Toko II, Kestemont P. 2012. État des lieux de la contamination des écosystèmes aquatiques par les pesticides organochlorés dans le Bassin cotonnier béninois. Cahiers Agricultures 21: 46-56. DOI:10.1684/agr.2012.0535.

Ahouangninou C, Fayomi BE, Martin T. 2011. Évaluation des risques sanitaires et environnementaux des pratiques phytosanitaires des producteurs maraîchers dans la commune rurale de Tori-Bossito (Sud-Bénin). Cahiers Agricultures 20 (3): 216-222. DOI:10.1684/ agr.2011.0485.

Belhadi A, Mehenni M, Reguieg L, Yakhlef H. 2016. Pratiques phytosanitaires des serristes maraîchers de trois localités de l'est des Ziban et leur impact potentiel sur la santé humaine et 1'environnement. Revue Agriculture 1(numéro spécial): 9-16.

Cissé I, Fall ST, Badiane M, Diop Y, Diouf A. 2006. Horticulture et usage des pesticides dans la zone des Niayes au Sénégal. Document de travail Écocité no 8. ISRA/LNERV, EISMV, LACT/Faculté de médecine pharmacie/UCAD. Disponible sur www.ecocite.org, $14 \mathrm{p}$.

Doumbia M, Kwadjo KE. 2009. Pratiques d'utilisation et de gestion des pesticides par les maraîchers en Côte d'Ivoire : cas de la ville d'Abidjan et deux de ses banlieues (Dabou et Anyama). Journal of Applied Biosciences 18: 992-1002.

FAO, OIT. 2013. Protégeons nos enfants des pesticides. Guide visuel d'animation. Disponible sur http://www.fao.org/docrep/019/ i3527f/i3527f.pdf.

FAOSTAT. 2016. Situation de la production de tomates au Burkina Faso. Disponible sur http://www.fao.org/faostat/fr/\#data/QC.

Gnankiné O, Mouton L, Savadogo A, Martin T, Sanon A, Dabire RK, et al. 2013. Biotype status and resistance to neonicotinoids and carbosulfan in Bemisia tabaci (Hemiptera: Aleyrodidae) in Burkina Faso, West Africa. International Journal of Pest Management 59 (2): 95-102. DOI:10.1080/09670874.2013.771806.

Guichard L, Dedieu F, Jeuffroy MH, Meynard JM, Reau R, Savini I. 2017. Le plan Ecophyto de réduction d'usage des pesticides en France : décryptage d'un échec et raisons d'espérer. Cahiers Agricultures 26(1): 14002. DOI:10.1051/cagri/2017004.

Houndété TA, Kétoh GK, Hema OSA, Brévault T, Glitho IA, Martin T. 2010. Insecticide resistance in field populations of Bemisia tabaci (Hemiptera: Aleyrodidae) in West Africa. Pest Management Science 66: 1181-1185. DOI:10.1002/ps.2008.

IFDC. 2007. Problématique de l'utilisation des produits phytosanitaires en conservation des denrées alimentaires et en maraîchage urbain et péri-urbain au Burkina Faso : cas de Bobo Dioulasso, Ouahigouya et Ouagadougou. Ouagadougou/Burkina Faso : IFDC. $51 \mathrm{p}$.

Kanda M, Djaneye-Boundjou G, Wala K, Gnandi K, Batawila K, Sanni A, et al. 2013. Application des pesticides en agriculture maraîchère au Togo. VertigO - la revue électronique en sciences de l'environnement 13 (1): 1-17.

Lehmann E, Oltramare C, Nfon DJJ, Konaté Y, De Alencastro LF. 2016a. Assessment of occupational exposure to pesticides with multi-class pesticide residues analysis in human hairs using a modified QuEChERS extraction method, case study of gardening areas in Burkina Faso. In: Annual Meeting of the International Association of Forensic Toxicologists (TIAFT), Brisbane, Australia.
Lehmann E, Morgan Fargues M, Congo N, Konaté Y, De Alencastro LF. 2016b. Pesticide application in gardening: assessment of resulting impact on water resources quality using grab samples and pocis, case study of Loumbila lake, Burkina Faso. In: 9th European Conference on Pesticides and Related Organic Micropollutants in the Environment (Santiago de Compostela).

Martin T, Ochou OG, Djihinto A, Traoré D, Togola M, Vassal JM, et al. 2005. Controlling an insecticide-resistant bollworm in West Africa. Short communication. Ecosystems and Environment 107: 409-411. DOI:10.1016/j.agee.2004.11.006.

Multigner L. 2005. Effets retardés des pesticides sur la santé humaine. Environnement, Risques \& Santé 4 (3): 187-194.

Naré RWA, Savadogo PW, Gnankambary Z, Nacro HB, Sedogo PM. 2014. Effect of three pesticides on soil dehydrogenase and fluorescein diacetate activities in vegetable garden in Burkina Faso. Current Research Journal of Biological Sciences 6 (2): 102-106.

Naré RWA, Savadogo PW, Gnankambary Z, Nacro HB, Sedogo MP. 2015. Analyzing risks related to the use of pesticides in vegetable gardens in Burkina Faso. Agriculture, Forestry and Fisheries 4(4): 165-172. DOI:10.11648/j.aff.20150404.13.

Ngom S, Traore S, Thiam MB, Anastasie M. 2012. Contamination des produits agricoles et de la nappe phréatique par les pesticides dans la zone des Niayes au Sénégal. Rev Sci Technol, Synthèse 25: 119-130.

Roditakis E, Vasakis E, Grispou M., Stavrakaki M, Nauen R, Gravouil M, et al. 2015. First report of Tuta absoluta resistance to diamide insecticides. Journal of Pest Science 88: 9-16. DOI:10.1007/ s10340-015-0643-5.

Samuel O, Saint-Laurent L. 2001. Guide de prévention pour les utilisateurs de pesticides en agriculture maraîchère. Québec : Institut de recherche en santé et en sécurité du travail du Québec (IRSST) Press. 92 p.

Schiffers B, Mar A. 2011. Sécurité des opérateurs et bonnes pratiques phytosanitaires. Manuel $n^{\circ} 4$ : COLEACP/PIP Press. Bruxelles/ Belgique : Programme PIP/COLEACP. 246 p.

Tarla DN, Meutchieye F, Assako VA, Fontem DA, Kome JJA. 2013. Exposure of market gardeners during pesticide application in the western highlands of Cameroon. Scholarly Journal of Agricultural Science 3 (5), 172-177.

Tarla DN, Manu IN, Tamedjouong ZT, Kamga A, Fontem DA. 2015. Plight of pesticide applicators in Cameroon: case of tomato (Lycopersiconesculentum Mill). Farmers in Foumbot. Journal of Agriculture and Environmental Sciences 4(2): 87-98. DOI: 10.15640/jaes.v4n2a10.

Toé AM. 2010. Étude pilote des intoxications dues aux pesticides agricoles au Burkina Faso. Secrétariat de la Convention de Rotterdam, 94 p. Disponible sur : www.pic.int/Portals/5/download. aspx?d=UNEP-FAO-RC-SHPFs-PilotstudyBurkina.Fr.pdf.

Tyagi H, Gautam T, Prashar P. 2015. Survey of pesticide use patterns and farmers' perceptions: a case study from cauliflower and tomato cultivating areas of district Faridabad, Haryana, India. International Journal of MediPharm Research 01 (03): 139-146.

Wognin AS, Ouffoue SK, Assemand EF, Tano K, Koffi-Nevry R. 2013. Perception des risques sanitaires dans le maraîchage à Abidjan, Côte d'Ivoire. International Journal of Biological and Chemical Sciences 7(5): 1829-1827. DOI: 10.4314/ijbcs.v7i5.4.

Citation de l'article : Son D, Somda I, Legreve A, Schiffers B. 2017. Pratiques phytosanitaires des producteurs de tomates du Burkina Faso et risques pour la santé et l'environnement. Cah. Agric. 26: 25005. 